\title{
On divided-difference semigroups
}

\author{
D. G. FitzGerald
}

December 21, 2009

\begin{abstract}
The Newtonian divided-difference operators generate the nil-Coxeter algebra and semigroup. A bijective correspondence between the nil-Coxeter semigroup and the symmetric group is used to provide braid-like diagrams for the former, and corresponding Reidemeister-type moves for the relations. Conditions are given for similar relations to hold in a skew group ring. Interesting extensions of the nil-Coxeter semigroup are described and given diagrammatic representations.

Keywords: divided-differences, nil-Coxeter algebra, Bruhat order, diagram semigroups, presentations.
\end{abstract}

\section{Introduction}

\subsection{Newton's divided-difference operators and the Nil-Coxeter semigroup relations}

Let $K$ be a field. The defining action of the symmetric group $\mathbb{S}_{n}$ on $\mathbf{n}=\{1,2, \ldots n\}$ induces actions on the vector space $V=K^{n}$ and on the field of rational functions $K\left[z_{1}, z_{2}, \ldots z_{n}\right]$. We write these as left actions defined, for $\pi \in \mathbb{S}_{n}$, by

$$
\begin{array}{cl}
\pi: i \mapsto \pi i & (i \in \mathbf{n}), \\
\pi \mathbf{z} & =\left(z_{\pi i}\right) \quad\left(\mathbf{x}=\left(z_{i}\right) \in V\right), \\
\pi f(\mathbf{z}) & =f(\pi \mathbf{z}) \quad(f \in K[\mathbf{z}]) ;
\end{array}
$$

$K$ is the fixed field, and the action on the polynomial ring $K(\mathbf{z})$ is a sub-act.

$\mathbb{S}_{n}$ is generated by transpositions $\sigma_{i j}=(i j),(i, j=1 \ldots n-1)$, in fact by the simple transpositions $\sigma_{i}=\sigma_{i, i+1}$. Define $\partial_{i j}: K[\mathbf{z}] \longrightarrow K[\mathbf{z}]$ by

$$
\partial_{i j} f=\frac{f-\sigma_{i j} f}{z_{i}-z_{j}}
$$

and denote by $\partial_{i}$ the simple divided-difference $\partial_{i, i+1}$. By the factor theorem, the $\partial_{i j}$ map $K(\mathbf{z}) \longrightarrow K(\mathbf{z})$. As operators, $\partial_{i j}=\left(z_{i}-z_{j}\right)^{-1}\left(1-\sigma_{i j}\right)$, and so they interact with $\sigma_{i j}$ according to

$$
\sigma_{i j} \partial_{i j}=\partial_{i j}, \quad \partial_{i j} \sigma_{i j}=-\partial_{i j}
$$


since, for any permutation $\pi, \pi \sigma_{i j} \pi^{-1}=\sigma_{\pi i, \pi j}$, we also have

$$
\pi \partial_{i j} \pi^{-1}=\left(z_{\pi i}-z_{\pi j}\right)^{-1} \pi\left(1-\sigma_{i j}\right) \pi^{-1}=\partial_{\pi i, \pi j} .
$$

Moreover, $\partial_{i j}$ is a $\left(1, \sigma_{i j}\right)$-derivation in that

$$
\partial_{i j} f g=f\left(\partial_{i j} g\right)+\left(\partial_{i j} f\right)\left(\sigma_{i j} g\right)=\left(\partial_{i j} f\right) g+\left(\sigma_{i j} f\right) \partial_{i j} g
$$

Importantly for our concerns here, the $\partial_{i}$ satisfy, for all $i, j \in\{1, \ldots, n-1\}$, the relations

$$
\begin{aligned}
\partial_{i}^{2} & =0 \\
\partial_{i} \partial_{i+1} \partial_{i} & =\partial_{i+1} \partial_{i} \partial_{i+1}, \\
\partial_{i} \partial_{j} & =\partial_{j} \partial_{i} \quad(|i-j|>1),
\end{aligned}
$$

reminiscent of those obtaining for the simple transpositions $\sigma_{i}=(i i+1)$ which generate the symmetric group in its presentation as a Coxeter group of type $A_{n-1}$. The algebra generated by these $\partial_{i}$ is thus called the nil-Coxeter algebra of type $A_{n-1}$; logically the semigroup $N D_{n}$ presented by the generators and relations given above could be called the nil-Coxeter semigroup of type $A_{n-1}$.

In the single-variable case, these operators have a distinguished history in an idea of Newton's: given data $(\mathbf{x}, \mathbf{y})$ with $\mathbf{y}=f(\mathbf{x})$, we can sequentially fit interpolating polynomials $p_{k}(x)$ of degree $k$ by

$$
p_{k}(x)=c_{0}+c_{1}\left(x-x_{1}\right)+c_{2}\left(x-x_{1}\right)\left(x-x_{2}\right)+\cdots
$$

such that $c_{k}$ can be calculated without recomputing the $c_{j}$ of lower degree terms. The coefficients are given by

$$
\begin{aligned}
& c_{0}=f\left(x_{1}\right), \\
& c_{1}=\left(x_{1}-x_{2}\right)^{-1}\left(y_{1}-y_{2}\right)=\partial_{1} f\left(x_{1}\right), \\
& c_{2}=\left(x_{2}-x_{3}\right)^{-1}\left(y_{2}-y_{3}\right)=\partial_{2} f\left(x_{2}\right), \quad \text { etc. },
\end{aligned}
$$

in notation which uses the action of $\mathbb{S}_{n}$ on $(\mathbf{x}, \mathbf{y})$. So $N D_{n}$ may also referred to as the Newton's or lesser divided-difference semigroup on $n$ points, because we study it here as a member of a suite of related semigroups arising from divided-difference operators.

\subsection{Coxeter groups, reduced words, Bruhat order.}

However the story really begins with a Coxeter group $G$ given by the presentation $\langle X \mid R\rangle \cong X^{*} / R^{\sharp}$, where $R^{\sharp}$ is the congruence generated by $R$. For $G$, we take $X=$ $\left\{x_{1}, x_{2}, \ldots x_{n-1}\right\}$ and $R=R_{G}=\left\{\left(x_{i} x_{j}\right)^{m_{i j}}=1\right\}$, where $\left(m_{i j}\right)$ is a symmetric $(n-1) \times$ $(n-1)$ matrix with all $m_{i i}=1$. It is convenient notation to define $(a b]^{n}$ to mean the initial segment of $(a b)^{n}$ of length $n$; thus $(a b]^{3}=a b a$, etc. Then the relations $R$ may be expressed as

$$
\begin{aligned}
x_{i}^{2} & =1 \\
\left(x_{i} x_{j}\right]^{m_{i j}} & =\left(x_{j} x_{i}\right]^{m_{i j}} \quad(i<j),
\end{aligned}
$$


in a form showing that (3) preserves the length of words in $X^{*}$. The values of $m_{i j}$ are conventionally encoded in a Coxeter graph; the symmetric group has $m_{i, i+1}=3$ and $m_{i j}=2$ for $|i-j|>1$. The words of minimum length in their $R^{\sharp}$-classes are called reduced words, and reduced words are related by transitions of the type (3). Any reduced word $R^{\sharp}$-equivalent to $w$ may be denoted by $[w]$. The length $\ell(w)$ of a member of $G$ is the length of any of its reduced words; $\ell(u v) \leq \ell(u)+\ell(v)$, with equality if and only if $u v$ is already reduced (i.e., $u v$ cannot be shortened by transitions of the kind (2)). There is a unique word $w_{0}$ of greatest length; in $\mathbb{S}_{n}, \ell\left(w_{0}\right)=n(n-1) / 2$.

The weak Bruhat order $\leq_{l}$ on $G$ is the transitive closure of the covering relation, $u \prec v$ if and only if $x_{i} u=v$ and $\ell(u)+1=\ell(v)$. The strong Bruhat order on $G$, denoted $\leq_{s}$, is characterised (in [6], Ex. 2.1.8) by

$$
u \leq_{s} v \Longleftrightarrow v=w_{1} u w_{2} \text { and } \ell(u) \leq \ell(v)
$$

for some $w_{1}, w_{2} \in G$. See also [5] for the Bruhat orders on $\mathbb{S}_{n}$; they are of considerable importance in the combinatorial study of permutations.

\subsection{Modifications; bijections}

If we modify the relations for $\mathbb{S}_{n}$ by retaining (3) but replacing (2) by

$$
x_{i}^{2}=x_{i},
$$

we get an idempotent-generated monoid $M_{n}$ (or a semigroup, depending on the ambient variety); alternatively if we modify (2) to

$$
x_{i}^{2}=0
$$

we get a nilpotent-generated semigroup $N D_{n}$ in the variety of semigroups with zero. The corresponding sets of relations and congruences may be subscripted for disambiguation, thus: $R_{S}, R_{M}, R_{N}$. Transitions in $R_{S}$ between reduced words may be chosen to be all of the type (3). So if $u, v$ are reduced words, then $u R_{S}^{\sharp} v$ if and only if $u R_{N}^{\sharp} v$ if and only if $u R_{M}^{\sharp} v$; the $R^{\sharp}$-classes differ on non-reduced words, of course. But this means that the non-zero, non-identity elements of the three semigroups are in bijective correspondence; this extends to bijections between $N D_{n}, \mathbb{S}_{n}$, and $M_{n}$ by associating $0,1_{S}$ and $1_{M}$. These bijections are not of course isomorphisms, but any representation of $\mathbb{S}_{n}$, for example by diagrams, transfers to a representation of $N D_{n}$ or $M_{n}$. Only the multiplication rule has to be changed!

Divided-difference operators and their properties have been discussed extensively in the literature; in addition to references above, see [1], [2], [3] and [4]. As well, see [7], [10] for informal literature on the idempotent variant (5).

Before returning to consequences of these modified relations, concentrating on nilpotentgenerated divided-difference semigroups, we detour to establish that they can be realised in certain operator-type rings. From now on, we consider only type $A$ Coxeter groups, i.e. symmetric groups (so $m_{i, i+1}=3$ and $m_{i j}=2$ for $|i-j|>1$ ). 


\section{Realisation in skew group rings}

Let the symmetric group $\mathbb{S}_{n} \cong\left\langle s_{i}\right\rangle_{i=1}^{n-1}$ act (on the left) on a field $F$, and let $F \mathbb{S}_{n}$ be the corresponding skew group ring, to which the action extends by linearity and

$$
s_{j}\left(a_{i} s_{i}\right)=\left(s_{j} a_{i}\right) s_{j} s_{i}
$$

See e.g. [8] for background on skew group rings.

Let us seek elements $t_{i} \neq 0$ of $F \mathbb{S}_{n}$, one for each $i$, satisfying the braid relations

$$
\begin{aligned}
t_{i} t_{i+1} t_{i} & =t_{i+1} t_{i} t_{i+1}, \\
t_{i} t_{j} & =t_{j} t_{i} \quad(|i-j|>1),
\end{aligned}
$$

and with the reasonable side condition (which we dub "embeddability") that these relations be preserved under the embeddings of any $\mathbb{S}_{m}$ in $\mathbb{S}_{n}(m<n)$ which result from the natural order-preserving embeddings of $\mathbf{m}$ in $\mathbf{n}$. For example, $\mathbb{S}_{2}$ embeds in $\mathbb{S}_{n}$ by each map $s_{1} \mapsto s_{i}$ for $i=1, \ldots n-1$, and $t_{1} \in F\left\langle s_{1}\right\rangle$, so $t_{1}=a_{1} 1+b s_{1}$; thus

$$
t_{i}=a_{i} 1+b_{i} s_{i}
$$

i.e., $t_{i} \in F\left\langle s_{i}\right\rangle$, which we assume throughout.

We say that $t_{i}$ has the symmetrising property if

$$
s_{i} t_{i}=t_{i}
$$

which is equivalent to each of $s_{i} a_{i}=b_{i}$ or $s_{i} b_{i}=a_{i}$. The first proposition deals with the connection between (10) and a generalisation of the "squaring" relations (5) and (6).

Proposition $1 t_{i}$ is symmetrising if and only if $t_{i}^{2}=\left(a_{i}+b_{i}\right) t_{i}$.

Proof. From (9) we have

$$
t_{i}^{2}=\left(a_{i}^{2}+b_{i}\left(s_{i} b_{i}\right)\right) 1+\left(a_{i} b_{i}+b_{i}\left(s_{i} a_{i}\right)\right) s_{i}
$$

while

$$
\left(a_{i}+b_{i}\right) t_{i}=\left(a_{i}^{2}+a_{i} b_{i}\right) 1+\left(a_{i} b_{i}+b_{i}^{2}\right) s .
$$

So these are equal if and only if $s_{i} a_{i}=b_{i}$.

It is also easy to see that $t_{i} t_{j}=t_{j} t_{i}$ is equivalent to $s_{i} s_{j}=s_{j} s_{i}, s_{i} a_{j}=a_{j}$ (and so, if $t_{i}$ is symmetrising, to $s_{i} b_{j}=b_{j}$ and to $s_{i} t_{j}=t_{j}$ ). So (8) holds in $F \mathbb{S}_{n}$. Let us next turn to the braid relations $(7)$.

Proposition 2 If each $t_{i}$ is symmetrising, then (7) is equivalent to the conjunction of

$$
\begin{aligned}
s_{i+1} a_{i} & =s_{i} a_{i+1} \quad\left(=c_{i}, \text { say }\right), \\
a_{i}+b_{i} & =q \in F, \text { with } s_{i} q=q, \text { and } \\
a_{i} a_{i+1} & =c_{i}\left(a_{i}+a_{i+1}-q\right) .
\end{aligned}
$$


Proof. By expanding (7), equating coefficients, and cancelling with the use of $s_{i} a_{i}=$ $b_{i} \neq 0$, we come to

$$
\begin{aligned}
a_{i} a_{i+1} a_{i}+b_{i}\left(s_{i} a_{i+1}\right) a_{i} & =a_{i+1} a_{i} a_{i+1}+b_{i+1}\left(s_{i+1} a_{i}\right) a_{i+1}, \\
a_{i} a_{i+1}+b_{i}\left(s_{i} a_{i+1}\right) & =a_{i+1}\left(s_{i} a_{i+1}\right), \\
a_{i+1} a_{i}+b_{i+1}\left(s_{i+1} a_{i}\right) & =a_{i}\left(s_{i+1} a_{i}\right) \\
s_{i} s_{i+1} a_{i} & =a_{i+1} \\
a_{i} & =s_{i+1} s_{i} a_{i+1} \\
b_{i}\left(s_{i} b_{i+1}\right)\left(s_{i} s_{i+1} b_{i}\right) & =b_{i+1}\left(s_{i+1} b_{i}\right)\left(s_{i+1} s_{i} b_{i+1}\right) .
\end{aligned}
$$

From $\left(\left[s s^{\prime}\right]\right)$ or the equivalent $\left(\left[s^{\prime} s\right]\right)$ we have (11). Now from $([s])$ and $\left(\left[s^{\prime}\right]\right)$ we have $a_{i} a_{i+1}=\left(s_{i} a_{i+1}\right)\left(a_{i+1}-b_{i}\right)=\left(s_{i+1} a_{i}\right)\left(a_{i}-b_{i+1}\right)$ and so $a_{i+1}-b_{i}=a_{i}-b_{i+1}$, i.e., $a_{i}+b_{i}=a_{i+1}+b_{i+1}$ for all $i$. Thus $q=a_{i}+b_{i}$ is also independent of $i$. Moreover, for all $i, s_{i} q=s_{i} a_{i}+s_{i} b_{i}=b_{i}+a_{i}=q$, so $q$ is in the fixed field of the action of $\mathbb{S}_{n}$. So (12) holds. Returning to $([s])$ we have

$$
a_{i} a_{i+1}=c_{i}\left(a_{i+1}-\left(q-a_{i}\right)\right),
$$

giving (13).

Conversely, we can check by reversing these computations that (10), (11), (12) and (13) imply the coefficient equations $([s])$ to $\left(\left[s^{\prime} s\right]\right)$. Also from (13) we have

$$
\begin{aligned}
a_{i} a_{i+1}\left(a_{i}-a_{i+1}\right) & =c_{i}\left(a_{i}+a_{i+1}-q\right)\left(a_{i}-a_{i+1}\right)=c_{i}\left(a_{i}-b_{i+1}\right)\left(a_{i}-a_{i+1}\right) \\
& =c_{i}\left(a_{i}\left(b_{i+1}-b_{i}\right)-b_{i+1}\left(a_{i}-a_{i+1}\right)\right),
\end{aligned}
$$

using $a_{i}-a_{i+1}=b_{i+1}-b_{i}$ from (12); so

$$
a_{i} a_{i+1}\left(a_{i}-a_{i+1}\right)=c_{i}\left(-a_{i} b_{i}+b_{i+1} a_{i+1}\right)
$$

which gives ([1]). Finally

$$
s_{i} s_{i+1} b_{i}=s_{i} s_{i+1} s_{i} a_{i}=s_{i+1} s_{i} s_{i+1} a_{i}=s_{i+1} a_{i+1}=b_{i+1},
$$

giving $\left(\left[s s^{\prime} s\right]\right)$.

Thus we have a semigroup inside $F \mathbb{S}_{n}$ which is generated by $\left\{q 1, t_{1}, t_{2}, \ldots\right\}$ and satisfies $(7,8)$ and a common generalisation of $(5)$ and $(6)$,

$$
t_{i}^{2}=q t_{i}, \text { with } q=\mathbb{S}_{n} q \in F .
$$

In particular, with $q=1,0$ we recover idempotent, resp. nilpotent, generators.

Corollaries. (i) In the case where $F=K\left(z_{1}, z_{2}, \ldots z_{n}\right)$, the choices $a_{i}=-b_{i}=$ $\left(z_{i}-z_{i+1}\right)^{-1}$ satisfy (11) and (12) with $q=0$, hence also $c_{i}^{-1}=a_{i}^{-1}+a_{i+1}^{-1}=\left(z_{i}-z_{i+2}\right)$, which is (13). This gives (returning to the use of $\sigma_{i}$ for generators) $t_{i}=\left(z_{i}-z_{i+1}\right)^{-1}\left(1-\sigma_{i}\right)=$ $\partial_{i}$.

(ii) For a solution with $q=1$, we take $a_{i}=z_{i}\left(z_{i}-z_{i+1}\right)^{-1}$ and $b_{i}=s_{i} a_{i}=$ $-z_{i+1}\left(z_{i}-z_{i+1}\right)^{-1}$. So (11) is met with $c_{i}=z_{i}\left(z_{i}-z_{i+2}\right)^{-1}$, and we may calculate that

$$
a_{i}^{-1}+a_{i+1}^{-1}-\left(a_{i} a_{i+1}\right)^{-1}=1-\frac{z_{i+2}}{z_{i}}=c_{i}^{-1},
$$


giving (13). Then

$$
\begin{aligned}
t_{i} & =z_{i}\left(z_{i}-z_{i+1}\right)^{-1} 1-z_{i+1}\left(z_{i}-z_{i+1}\right)^{-1} \sigma_{i} \\
& =\left(z_{i}-z_{i+1}\right)^{-1}\left(z_{i} 1-z_{i+1} \sigma_{i}\right)=\left(z_{i}-z_{i+1}\right)^{-1}\left(1-\sigma_{i}\right) z_{i} 1=\partial_{i} z_{i} 1,
\end{aligned}
$$

which is the isobaric divided-difference operator.

(iii) Similarly, a third solution is given by $a_{i}=z_{i+1}\left(z_{i}-z_{i+1}\right)^{-1}$ and $b_{i}=s_{i} a_{i}=$ $-z_{i}\left(z_{i}-z_{i+1}\right)^{-1}$, thus with $a_{i+1}=z_{i+2}\left(z_{i+1}-z_{i+2}\right)^{-1}, c_{i}=z_{i+2}\left(z_{i}-z_{i+2}\right)^{-1}$ and $q=$ -1 .

These particular solutions are noted by Lascoux and Schützenberger [4]. See also [1] or [2]. The "symmetric part" operator, also idempotent, ev $f:=\frac{1}{2}\left(1+\sigma_{i}\right) f$, is of the form (9) with $a_{i}=b_{i}=\frac{1}{2}$. It does not satisfy (7) but is related to the isobaric divided-difference operator, by

$$
\begin{aligned}
\partial_{i}\left(z_{i} f\right) & =\partial z_{i} \cdot f+\sigma_{i} z_{i} \cdot \partial f=f+z_{i+1} \cdot \partial_{i} f \\
& =z_{i} \cdot \partial_{i} f+\partial_{i} z_{i} \cdot \sigma_{i} f=\sigma_{i} \cdot f+z_{i} \partial_{i} f \\
& =\frac{1}{2}\left(1+\sigma_{i}\right) f+\frac{1}{2}\left(z_{i}+z_{i+1}\right) \partial_{i} f=\operatorname{ev} f+\mathrm{ev} z_{i} \cdot \partial_{i} f .
\end{aligned}
$$

\section{Diagram semigroups and moves}

For present purposes, though the concept is much broader, a diagram is a graph with $n$ vertices, aligned in a $2 \times n$ matrix, and $n$ edges (or strands) each incident on one vertex of the top row and one of the bottom row. The graph is drawn in the plane, with crossings allowed, and the edges are marked or decorated to show crossings of various kinds. Homotopy-equivalent drawings are regarded as representing the same diagram. There is also a notion of moves, (which may embody homotopy equivalence in a larger ambient space, for example) and which (together with homotopy equivalence) establish an equivalence relation, denoted by $\sim$, on diagrams. (Such moves mimic the familiar Reidemeister moves of knot theory, and are usually drawn so that irrelevant strands are omitted.)

Diagrams $u$ and $v$ (say) are concatenated by the following steps: (i) juxtaposing diagrams for $u$ and $v$, with the left factor $u$ above; (ii) identifying the lower row of vertices in $u$ with the upper row of vertices in $v$; (iii) erasing these "interior" points; and then (iv) simplifying the composite diagram according to the given moves, resulting in another diagram. The concatenations $u(v w)$ and $(u v) w$ are homotopy- and $\sim$-equivalent, and so the $\sim$-classes form a semigroup, which we call a diagram semigroup.

A standard example is provided by a representation $\mathcal{S}_{n}$ of the symmetric group. Refer to Fig. 1, which shows schematically the diagram which is the image of $s_{i}$, one of the standard generators in the Coxeter presentation, and the product $s_{i} s_{i+1}$ in $S_{5}$. Edges join vertices $\pi i$ (in an upper row) and $i$ (in the lower row); note the reversal of the usual order since we are treating these as left mappings. Here they will be drawn with braid-like crossings, that is, as a projection of a braid, but it will be irrelevant whether one strand crosses another "above" or "below". See also Fig.2, which shows the moves for $S_{n}$. 

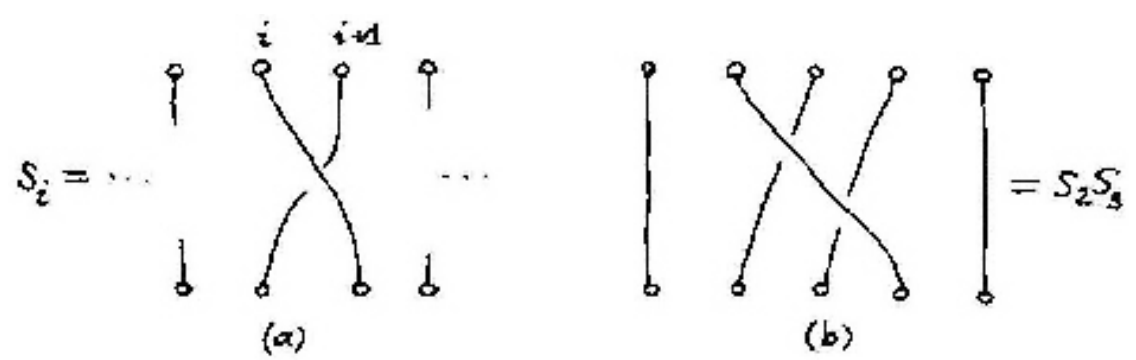

Figure 1: Diagrams for $S_{n}$ : (a) the generator $s_{i}$; (b) an example of a product in $S_{5}$.

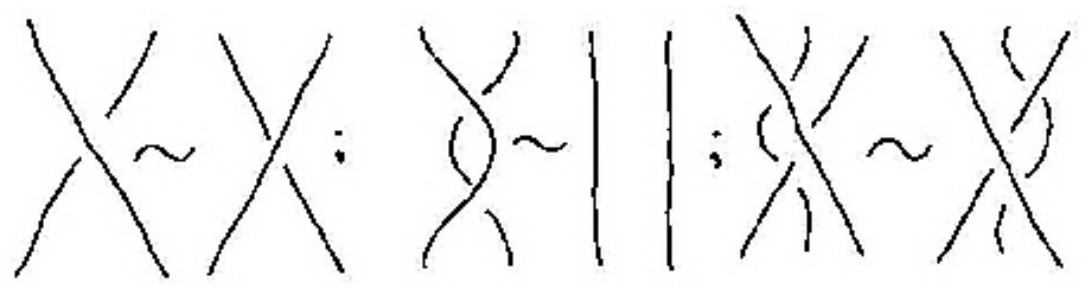

Figure 2: Moves for $S_{n}$.

\subsection{More on presentations}

Given a semigroup $T$, a set $X$, and $f: X \longrightarrow T$, write $f^{+}$for the unique homomorphism of $X^{+}$to $T$ which extends $f$. We say that relations $R \subseteq X^{+} \times X^{+}$are satisfied in $T$ via $f$ if for all $(u, v) \in R, f^{+}(u)=f^{+}(v)$, and as before write $R^{\sharp}$ for the congruence on $X^{+}$ generated by $R$. In this case, there is a unique canonical homomorphism $\phi:\langle X \mid R\rangle \longrightarrow$ $T$ such that $\left.\phi\right|_{X}=f ; \phi$ is surjective if and only if $f(X)$ is a set of generators for $T$. If $T$ is a diagram semigroup, we say $T$ has the slice property if every diagram in $T$ may be drawn so that a finite number of horizontal lines, each intersecting the $n$ strands, divides the diagram into layers such that each contains exactly one crossing. We say $T$ has the transition property if for any move $s \sim t$ in $T$, and any valid assignment of vertices to the strands, there are $u, v \in X^{+}$such that $f^{+}(u)=s, f^{+}(v)=t$, and $u$ and $v$ are connected by a sequence of elementary transitions in $R$. (Note that moves are diagrams of one or two layers in which vertices and irrelevant strands have been omitted, and thus represent collections of relations on "free" diagrams.) For a diagram semigroup $T$ we have by standard methods

Proposition 3 Let relations $R$ be satisfied in $T$ via $f: X \rightarrow T$, and let have the slice property. Then the canonical homomorphism $\phi$ is

(i) surjective if and only if each slice contains the image of a member of $X$; and

(ii) injective if and only if $T$ has the transition property.

Unfortunately this does not help to give a characterisation (e.g. geometric) of those diagrams which form the semigroup. At any rate, we will now be dealing with three kinds of semigroups, or rather, three levels of abstraction: at base, the level of operators 
as expressed in section 1.1; above, the level of abstract presentations, as in section 1.2; and intermediate, the level of diagrammatic representations. These are distinguished notationally by the use of different typefaces: latin for the presentations $\left(N D_{n}\right.$, etc.), calligraphic for the diagrams $\left(\mathcal{N D} \mathcal{D}_{n}\right.$, etc.) and blackboard-boldface for the "real" $\left(\mathbb{N}_{n}\right.$, etc.).

\section{The Newton's divided-difference semigroup}

We return to this semigroup, introduced in sections 1.1 and 1.3 ; it has presentation $\langle X \mid R\rangle$, with $X=\left\{x_{i}: i \in \mathbf{n}-\mathbf{1}\right\}$ and $R$ composed of the relations (6), (7) and (8). Because of the bijection between $S_{n}$ and $N D_{n}$ noted in section 1.3, the members of $N D_{n}$ may be represented by diagrams based on those for $S_{n}$. To distinguish the diagrams, we will use nodes on the crossings (drawn as filled circles) in place of the bypasses used for $S_{n}$. See Fig. 3 for the image of the generator $d_{i}$ and corresponding moves for $N D_{n}$.

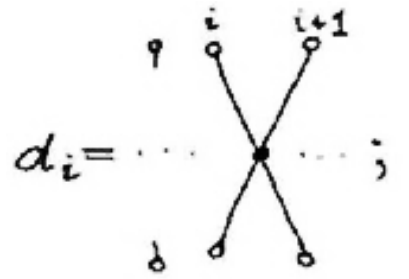

(a)

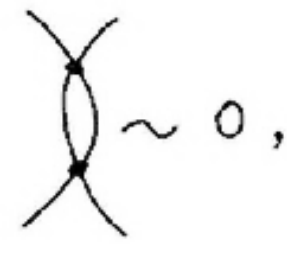

(b)

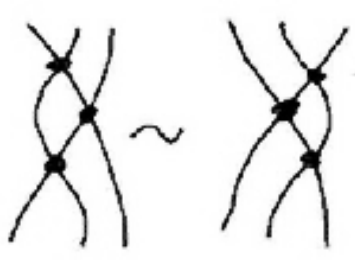

Figure 3: Diagrams and moves for $N D_{n}$ : (a) the generator $d_{i}$; (b) moves for $N D_{n}$.

Remark. We can produce a similar representation for the idempotent-generated monoid $M_{n}$, using (say) open circles at the crossings. The move shown in Fig. 4. replaces the first move in Fig. 3(b).

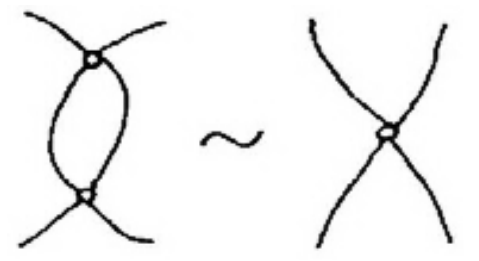

Figure 4: New move for $M_{n}$.

We may verify that the slice and transition properties hold in $\mathcal{L D}_{n}$, so the canonical map is an isomorphism, $L D_{n} \cong \mathcal{L D}_{n}$. The relations also hold in $\mathbb{L}_{n}$, so $\phi$ : $L D_{n} \longrightarrow \mathcal{L D}_{n}$ is surjective. In fact it is bijective, since the Schubert polynomial $\mathfrak{S}_{w_{0}}=z_{1}^{n-1} z_{2}^{n-2} \ldots z_{n-1}$ separates the members of $\mathbb{N D}_{n}$ by

$$
\partial_{w} \mathfrak{S}_{w_{0}}=\mathfrak{S}_{\left[w_{0} w^{-1}\right]}
$$

and the $\mathfrak{S}_{[w]}$ are distinct - in fact, a basis for $P_{n-1}([5],(4.13))$. 


\subsection{Ideal structure and orders}

$N D_{n}$ is self-dual under the reversal of words ( $R^{\sharp}$-equivalence is preserved because the words in (3) are symmetric under reversal). Denote by $\ell(u)$ the length function as described in section 1.2. So $\ell(u) \geq 1$ for all $u \neq 0$, and $\ell(u v)=\ell(u)+\ell(v)$ if $u v \neq 0$. Also observe that all non-reduced words in $N D_{n}$ are equivalent to the zero, and if $w$ is a reduced word, that $\ell(w)$ coincides with the (minimal) number of crossings in a diagram for $w$.

Proposition 4 Let $u, v \in N D_{n}$. Then $(u, v) \in \mathcal{R}$ if and only if $u=v$.

Proof. Suppose $(u, v) \in \mathcal{R}$. Then $u=0$ if and only if $v=0$, so suppose $u, v \neq 0$. Now $u=v$ or there exists $w \in N D_{n}$ such that $u=v w$, when $\ell(u)=\ell(v)+\ell(w)>\ell(v)$; similarly, $u=v$ or $\ell(v)>\ell(u)$. Hence the only possibility is that $u=v$. The reverse direction is trivial.

Hence the preorder $\leq_{R}$ is actually a (partial) order; by lateral duality, $\mathcal{L}$ is trivial and so also $\mathcal{J}$, and their associated preorders are really orders too. Define $\leq_{R}^{\prime}$ by

$$
u \leq_{R}^{\prime} v \text { if and only if } 0 \neq u \leq_{R} v \text { or } v=0
$$

That is, the bottom element in $\leq_{R}$ is moved to be the top element in $\leq_{R}^{\prime}$. Similarly we define $\leq_{J}^{\prime}$. The next proposition is immediate from the descriptions in section 1.2.

Proposition $\mathbf{5} \leq_{R}^{\prime}$ and $\leq_{J}^{\prime}$ are partial orders. Transferred to $\mathbb{S}_{n}$ they coincide respectively with the weak and strong Bruhat orders.

\section{The greater divided-difference semigroup and its signed extension}

Note that $\partial_{13}\left(z_{1}^{2} z_{2}\right)=z_{2}\left(z_{1}+z_{3}\right)$, which is symmetric with respect to neither $\sigma_{1}$ nor $\sigma_{2}$; thus $\partial_{13} \notin \mathbb{N D}_{3}$. By embeddability arguments, $\partial_{i j} \notin \mathbb{N D}_{n}$ for any $n \geq 3$ and $i \neq j \in \mathbf{n}$. This raises the question of studying the semigroup $\mathbb{G D}_{n}=\left\langle\partial_{i j} \mid i<j \in \mathbf{n}\right\rangle$. $\mathbb{N}_{n}$ is a subsemigroup, so (6), (7) and (8) still hold. Let $X=\left\{x_{i j} \mid i<j \in \mathbf{n}\right\}$ be a set of generators, and consider the relations

$$
\begin{aligned}
x_{i j}^{2} & =0, \text { all } i<j, \\
x_{i j} x_{k l} & =x_{k l} x_{i j} \text { for } i<j<k<l, \\
x_{i j} x_{j k} x_{i j} & =x_{j k} x_{i j} x_{j k}=x_{i j} x_{j k} x_{i k} \text { for } i<j<k .
\end{aligned}
$$

Now let $\pi$ be a permutation such that $\pi(1,2,3,4)=(i, j, k, l)$. Then $\partial_{i j}={ }^{\pi} \partial_{12}, \partial_{j k}=$ ${ }^{\pi} \partial_{23}, \partial_{k l}={ }^{\pi} \partial_{34}$ and moreover

$$
\begin{aligned}
\partial_{12} \partial_{23} \partial_{13} & =\partial_{1} \partial_{2} \sigma_{2} \partial_{1} \sigma_{2}=-\partial_{1} \partial_{2} \partial_{1} \sigma_{2} \\
& =-\partial_{2} \partial_{1} \partial_{2} \sigma_{2}=\partial_{2} \partial_{1} \partial_{2} .
\end{aligned}
$$


(These calculations take place in $\left\langle\mathbb{S}_{n}, \mathbb{N D}_{n}\right\rangle$.) It follows that the relations 15 to 17 , which we refer to collectively as $R_{G}$, hold in $\mathbb{G}_{n}$ via the map $x_{i j} \mapsto \partial_{i j}$. We may verify the slice and transition properties hold. It follows that there is a surjective homomorphism $\phi$ from $G D_{n}=\langle X \mid R\rangle$ to $\mathbb{G D}_{n}$. There is also an isomorphism from $G D_{n}$ to the semigroup $\mathcal{G D}_{n}$ of diagrams generated by the set $\left\{d_{i j} \mid i<j \in \mathbf{n}\right\}$ (where $d_{i j}$ is illustrated in Fig. $5(\mathrm{a})$ ), subject to previous moves together with those illustrated in Fig. 5(b). What is

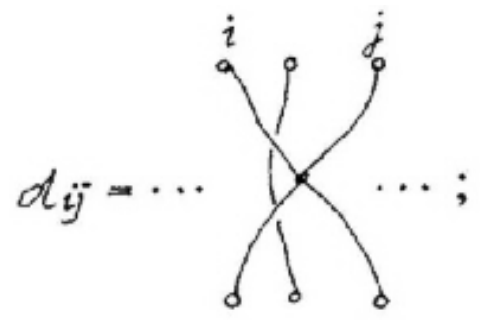

(a)

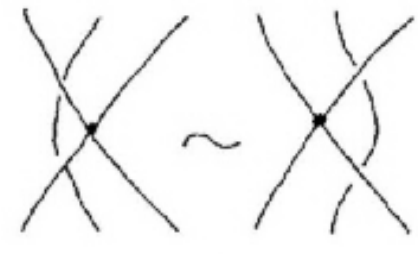

(b)

Figure 5: (a) Image of generator $d_{i j}$ in $\mathcal{G D}_{n}$; (b) extra move in $\mathcal{G D}_{n}$.

missing is a good geometric description of the diagrams which make up $\mathcal{G} \mathcal{D}_{n}$, and a proof or counterexample of the injectivity of $\phi: G D_{n} \longrightarrow \mathbb{G}_{n}$. Is there a set of polynomials which separate the members of $\mathbb{G}_{n}$ ? Perhaps the single Schubert polynomial $\mathfrak{S}_{w_{0}}$ still does so in $\mathbb{G D}_{n}$ as it does in $\mathbb{N D}_{n}$.

We may remark that the transition in Fig. 6 is a non-move, as (calculating in $\mathbb{G}_{n}$ ) $\partial_{13}\left(z_{1}^{2} z_{3}\right)=z_{1} z_{3}$ and $\partial_{1}\left(z_{1} z_{3}\right)=z_{3} \neq-z_{1}=\partial_{2}\left(z_{1} z_{3}\right)$, so $d_{1} d_{13} \neq \pm d_{2} d_{13}$ in $G D_{n}$.

Structure. Define a length function in $\mathbb{G}_{n}$ as the usual length of a reduced word; for $w$ reduced, $\ell(w)$ is also the number of nodes (not crossings) in the (reduced) diagram for $w$. Again we have, if $u v \neq 0$,

$$
\ell(u v)=\ell(u)+\ell(v),
$$

and the same argument as in Proposition 4 shows that $\mathbb{G}_{n}$ is $\mathcal{J}$-trivial.

There is another variant, the semigroup $\pm \mathbb{G}_{n}$ of signed divided-differences, in which (for $i<j$ ) $\partial_{j i}$ is identified with $-\partial_{i j}$. The relations for $\pm \mathbb{G}_{n}$ include:

$$
x_{i j}=-x_{j i} \text {, all } i \neq j,
$$

and we work in the variety of signed semigroups with zero, where there is a unary map $u \mapsto-u$ satisfying $-(-u)=u$ and $u(-v)=-u v$. Thus we may use generators $x_{i j}$ with

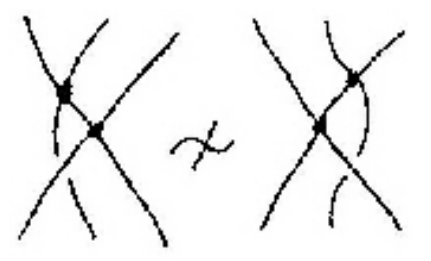

Figure 6: A non-move for $\mathcal{G D}_{n}$. 
$i \neq j,(18)$ is added, and the relations (15), (16) and (17) apply for $i \neq j$. The diagrams and moves are the same, but with minus signs included as appropriate to distinguish $d_{i j}$ and $d_{j i}$, etc.

\section{The permutation-divided-difference monoid}

The discussion in the previous section in turn suggests the investigation of the operator monoid $\mathbb{P D}_{n}=\left\langle\mathbb{S}_{n}, \mathbb{N D}_{n}\right\rangle$ which we call the permutation-divided-difference monoid. The relevant variety now is that of signed monoids with zero and we work with the generating set $\left\{\sigma_{i}, \partial_{i} \mid i \in \mathbf{n}-\mathbf{1}\right\}$. We revert to notation which uses $s_{i}$ and $d_{i}$ for generators in the abstract version.

$\mathbb{N D}_{n}$ and $\mathbb{S}_{n}$ are subsemigroups, so the relations (2) and (3) for the $s_{i}$, and (7) and (8) for the $d_{i}$, continue to hold via the mapping $s_{i} \mapsto \sigma_{i}, d_{i} \mapsto \partial_{i}$. As well, for all $i \in \mathbf{n}-\mathbf{1}$, the extra relations

$$
\begin{aligned}
s_{i} d_{i} & =d_{i}=-d_{i} s_{i}, \\
s_{i} d_{j} & =d_{j}=d_{j} s_{i} \text { for }|i-j| \geq 2, \text { and } \\
s_{i} d_{i+1} s_{i} & =s_{i+1} d_{i} s_{i+1},
\end{aligned}
$$

hold in $\mathbb{P D}_{n}$; we call the set of all these relations $R_{P D}$. The (real) permutation-divideddifference semigroup is thus a homomorphic image of $\left\langle s_{i}, d_{i} \mid R_{P D}\right\rangle=P D_{n}$. We define $\mathcal{P} \mathcal{D}_{n}$ to be the set of all diagrams with crossings either nodes or bypasses. The moves of $\mathcal{S}_{n}, \mathcal{N} \mathcal{D}_{n}$ and $\mathcal{G D}_{n}$ still hold, together with new ones shown in Fig. 7. Under composition of diagrams and use of these moves, $\mathcal{P} \mathcal{D}_{n}$ forms a semigroup, in fact a signed monoid with zero.
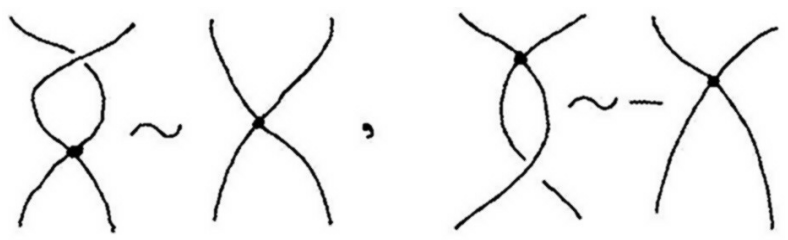

Figure 7: Extra moves for $\mathcal{P D}_{n}$.

Then relations $R_{P D}$ hold in $\mathcal{P} \mathcal{D}_{n}$ too, and, since the slice property also holds in $\mathcal{P} \mathcal{D}_{n}$, it is a homomorphic image of $\left\langle s_{i}, d_{i} \mid R_{P D}\right\rangle$. By inspection, the transition property holds, so $\mathcal{P} \mathcal{D}_{n} \cong P D_{n}$. Note that the group of units of $P D_{n}$ is $\pm S_{n}=\left\{\pi,-\pi: \pi \in S_{n}\right\}$. We may extend from $G D_{n}$ the definition of the length of a word in $P D_{n}$ as the number of $d$-factors in $w$.

Structure. We shall say a monoid $T$ is $G J^{1}$-factorisable if there are a subgroup $G$ and a $\mathcal{J}$-trivial subsemigroup $J$ such that $T=G J^{1}$.

Proposition 6 (i) if $w \in G D_{n}$, and $\pi \in \pm S_{n}$, then $\pi w \pi^{-1} \in G D_{n}$;

(ii) $P D_{n}$ is $G J^{1}$-factorisable, with $G= \pm S_{n}$ and $J=G D_{n}$. 
Proof. (i) Eqn. (1) confirms that the claim is true for words of length 1. Its truth for words of length $k$ implies that if $w$ has length $k+1$, so $w=\mu w_{1} \pi d_{i j} \rho$ with $\mu, \pi, \rho \in \pm S_{n}$ and $w$ of length $k$, then

$$
w=\mu w_{1} \pi d_{i j} \rho=\mu \pi \rho \cdot(\pi \rho)^{-1} w_{1} \pi \rho \cdot\left(\rho^{-1} d_{i j} \rho\right),
$$

and thus implies its truth for $k+1$.

(ii) Again by induction on the length of $a \in P D_{n}$. The inductive step relies on

$$
a_{1}=a d_{i} \rho \Longrightarrow a_{1}=\rho\left(\rho^{-1} a \rho\right)\left(\rho^{-1} d_{i} \rho\right),
$$

where $a, a_{1}$ have lengths $n$ and $n+1$.

\section{Summary}

So what we know about the relations between these semigroups may be summarised in this diagram:

$\begin{array}{cccccc}L D_{n} & \hookrightarrow & G D_{n} & \hookrightarrow & P D_{n} \\ \text { iso } \downarrow & & \text { iso } \downarrow & & \text { iso } \downarrow \\ \mathcal{L} \mathcal{D}_{n} & \hookrightarrow & \mathcal{G} \mathcal{D}_{n} & \hookrightarrow & \mathcal{P} \mathcal{D}_{n} \\ \text { iso } \downarrow & & ? l^{\prime} & & ? \downarrow \\ \mathbb{L} \mathbb{D}_{n} & \hookrightarrow & \mathbb{G}_{n} & \hookrightarrow & \mathbb{P D}_{n}\end{array}$

Clearly there is much to find out about their structure, combinatorics and relationships.

Acknowledgements. The author extends sincere thanks to Norman Do, Alex Molev, Stuart Margolis and Barry Gardner for pointing him to some of the literature, and to Jon McCammond for geometrical discussions.

\section{References}

[1] I. N. Bernšteĭn, I. M. Gel'fand, and S. I. Gel'fand, Schubert cells, and the cohomology of the spaces G/P. Uspehi Mat. Nauk 28 (1973), 3-26; Eng. trans. in Russian Math. Surveys 28 (1973), 1-26.

[2] Gutkin, E. A. Matrices that are connected with groups generated by reflections. (Russian) Funkcional. Anal. i Priložen. 7 (1973), no. 2, 81-82.

[3] A. Lascoux and M.-P. Schützenberger, Polynômes de Schubert, C. R. Acad. Sci. Paris, 294 (1982), 447-450.

[4] A. Lascoux and M.-P. Schützenberger, Décompositions dans l'algèbre des différences divisées, Discrete Math. 99 (1992), 165-179.

[5] I. G. Macdonald, Notes on Schubert polynomials, Publications du LACIM, No. 6, Université du Québec de Montréal, 1991. 
[6] L. Manivel, "Symmetric functions, Schubert polynomials and degeneracy loci", (trans. J. R. Swallow) SMF/AMS Texts and Monographs, American Mathematical Society, Providence, RI; Société Mathématique de France, Paris, 2001.

[7] S. Margolis, On some monoids associated to Coxeter groups, abstract of talk at North British Semigroups and Applications Network, 23rd July 2009, Heriot-Watt University, Edinburgh. Webpage: http://www.maths.manchester.ac.uk/ mkambites/ (accessed 20 Nov 2009).

[8] D. Passman, "Infinite crossed products", Pure \& Appl. Math. vol. 135, Academic Press, Inc., Boston, MA, 1989.

[9] B. E. Sagan, "The symmetric group. Representations, combinatorial algorithms, and symmetric functions", 2nd ed., Graduate Texts in Mathematics, vol. 203, Springer-Verlag, New York, 2001.

[10] T. Trimble, Buildings and BN-Pairs, notes dated March 19, 2007, webpage: http://www.math.ucr.edu/home/baez/trimble/ (accessed 20 Nov 2009).

School of Mathematics and Physics

University of Tasmania

Private Bag 37

Hobart 7001

Australia

email: D.FitzGerald@utas.edu.au 